\title{
Aniridia Intra-ocular Lens Implantation in a Geriatric Patient
}

The Editor,

Sir,

An 80-year old man was seen, due to visual loss bilaterally and a disquieting glare in his right-eye. In his medical history, he had a blunt trauma and underwent a primary penetrating surgery of his right-eye a year previously. In addition, the patient declared that he had chronic visual loss in his left-eye. Biomicroscopic examination of the right-eye revealed a relatively clear cornea, widely extended conjunctival scarring at superonasal and inferotemporal sides, vitreous prolapsus into the anterior chamber, aphakia with total absence of capsule and nearly 9 clock hours absent of the iris (Figure).

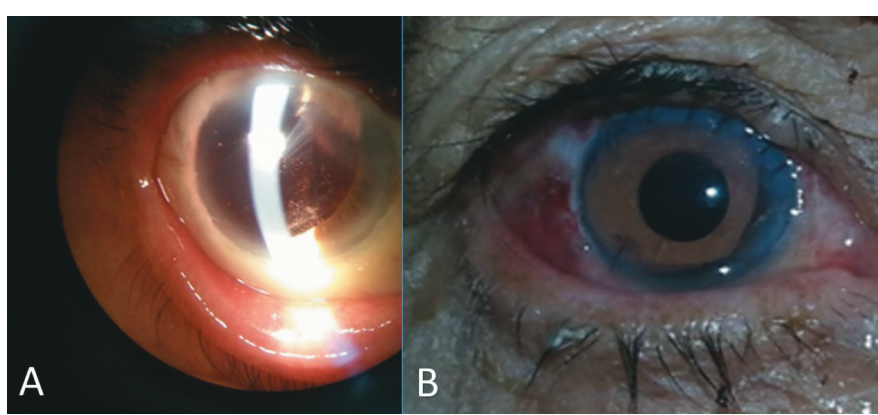

Figure: Pre-oprative image designates pre-operative vitreous prolapsus to the anterior chamber, aphakia with absent capsule and extensive iris defect (A). Image illustrates the postoperative appearance of the righteye with cosmetic improvement (B).

There was a mature cataract in his left-eye as well. His visual acuities were 20/1250 (4/10 with an aphakia correction of +11.0 ) and light perception in the right and left eyes, respectively. Overall, phacoemulsification was planned for his left-eye. After an uneventful surgery his best-corrected visual aquity (BCVA) was 20/20 after a small refractive correction of $-0.75-1.0 \times 110$ in his left-eye. Since the subtotal absence of the iris and aphakia with no remnant of the capsule, an aniridia intraocular lens (IOL) with scleral fixation was carried out on his right-eye (Ophtec 311, brown). Following the conjunctival peritomy, scleral flaps at 2 and 8 O'clock are made to cover the knots of the double-arm 10/0 prolen sutures.

The needle of the non-absorbable 10/0 prolen suture was passed through the scleral flap to another one. Superior corneal incision was enlarged to let the implication of $9 \mathrm{~mm}$ wideness of the aniridia lens optic. Trans-scleral fixation of the IOL was performed to $1 \mathrm{~mm}$ posterior to the limbus. Thereafter, scleral flaps were closed using 10/0 nylon suture. Conjunctival was closed by using a $8 / 0$ vicryl suture. Corneal incision was sutured continuously with $10 / 0$ nylon suture. At the first day visit, examination revealed a BCVA 20/28 with the correction of $2.50 \times 15$ refractive error without any complication. The right-eye was quite cosmetic compared with the left eye's colour (Fig. 2). The fundus could be readily seen through the $4 \mathrm{~mm}$ diameter pupil size of IOL optic.

Prosthetic iris devices (PID) have been previously established as an effective method for the treatment of congenital or acquired aniridia $(1,2)$. Many types of PIDs have been produced with respect to the severity and extent of the iris defect. Prosthetic iris devices can be classified as endocapsular PIDs, combined lens-iris diaphragm (aniridia lens) and particular artificial iris (2). Since this patient had extensive aniridia and aphakia with absence of the capsule, a combined lens-iris diaphragm has been used. Although being effective, the aniridia lenses need a large corneal incision for its placement, due to its rigid and unfoldable material [polymethylmethacrylate] $(1,2)$.

Accordingly, we would like to highlight that combined lens-iris diaphragm keeping in mind the complications could be readily used for the treatment of combined aniridia and aphakia with absence of capsule.

Keywords: Aniridia lens, aphakia, trauma

\section{S Helvaci}

From: Elbistan State Hospital, Department of Ophtalmology, Kahramanmaraş, Turkey.

Correspondence: S Helvacı, Elbistan Devlet Hastanesi, 46300 Kahramanmaraş, Turkey. Fax: +90 34441380 05. e-mail: sezerhelvaci1985@hotmail.com.

\section{REFERENCES}

1. Srinivasan S, Ting DS, Snyder ME, Prasad S, Koch HR. Prosthetic iris devices. Can J Ophthalmol 2014; 49: 6-17.

2. Forlini C, Forlini M, Rejdak R, Prokopiuk A, Levkina O, Bratu A et al. Simultaneous correction of post-traumatic aphakia and aniridia with the use of artificial iris and IOL implantation. Graefes Arch Clin Exp Ophthalmol 2013; 251: 667-75. 